\title{
Crowd4City: Utilizando Sensores Humanos como Fonte de Dados em Cidades Inteligentes
}

\author{
Ana Gabrielle Ramos Falcão, Cláudio de Souza Baptista, Luciana Cavalcante de \\ Menezes
}

Laboratório de Sistemas de Informação/Departamento de Sistemas e Computação
Universidade Federal Campina Grande (UFCG)
Campina Grande - PB - Brasil - http://www.lsi.dsc.ufcg.edu.br
\{baptista, luciana\}@dsc.ufcg.edu.br, anagabrielle@copin.ufcg.edu.br

\begin{abstract}
Smart Cities solutions are currently being studied and applied by researchers and governments throughout the world with the purpose of better managing the problems found with the increase of urban populations and negative impacts suffered by the nature. The majority of the existing proposed models, however, use digital sensors for the gathering of the necessary information for its execution, which may interfere directly with the feasibility of its application on real world scenarios. With the intention of avoiding these problems we here suggest the use of Crowd Sensors for Smart Cities systems, mention the prototype we will develop and focus our study on the analysis of Crowd Sensors in this context.
\end{abstract}

Resumo. Soluções de Cidades Inteligentes estão sendo estudadas e aplicadas por pesquisadores e governos por todo o mundo a fim de contornar os problemas encontrados com o crescimento das populações urbanas e pelos impactos negativos sofridos pela natureza. Grande parte dos modelos propostos na literatura, no entanto, utiliza sensores digitais para recuperação das informações necessárias para sua execução, o que pode inviabilizar sua aplicação em cenários reais. Para contornar este problema e atingir uma gama maior de informações, este artigo sugere o uso de Sensores Humanos nos sistemas de Cidades Inteligente e cita o protótipo a ser desenvolvido, focando o estudo na análise dos Sensores Humanos neste contexto.

\section{Introdução}

Atualmente, é estimado que cerca de $50 \%$ da população mundial vive em áreas urbanas (Naphade et al., 2011). Até o ano 2050, a Organização das Nações Unidas (ONU) prevê que este número chegue a $70 \%$ devido, principalmente, ao crescimento da atual população urbana e ao crescente êxodo rural causado pela mecanização dos serviços e consequente redução na necessidade de trabalho manual na agricultura.

O resultado desta inflação da população urbana são cidades cada vez mais densas, trazendo grandes desafios para o governo como questões de administração dos congestionamentos no tráfego, dificuldade de acesso a recursos básicos, maior marginalidade e mais altos índices de criminalidade. 
Além disso, o meio ambiente está sendo cada vez mais deteriorado apresentando índices de poluição e desmatamento maiores, utilização dos recursos naturais sem discriminação e alteração das condições climáticas regulares -, causando problemas em toda a fauna e flora e contribuindo para o desencadeamento de desastres naturais cada vez de maiores proporções. Assim, cresce a preocupação das autoridades e da população pela reversão destes impactos, preservação da natureza e manutenção do meio ambiente, tendo em vista um futuro mais promissor.

As autoridades das cidades, que possuem como objetivo a redução de seus custos e como obrigação dispor dos recursos básicos à população (como acesso à água tratada, energia, transporte, serviços de suporte à saúde, educação e segurança), deparam-se com a necessidade de novas técnicas de planejamento, design, financiamento, construção, governança e operação dos serviços e infraestrutura urbana. Uma abordagem sugerida para contornar estes problemas, cada vez mais utilizada por todo o mundo, é a transformação destas áreas urbanas em Cidades Inteligentes - Smart Cities.

A expressão "Cidades Inteligentes" começou a ser utilizada no final da década de 1990, porém, apenas recentemente (por volta de 2004) vem sendo utilizada com o emprego de sistemas de informação na integração da operação de serviços e infraestrutura urbana. O objetivo principal destes sistemas é disponibilizar tais serviços, todo o tempo, a toda a população de uma maneira aprimorada, com maior eficiência, balanceando os custos e usando os recursos naturais de forma a agredir o meio ambiente o mínimo possível.

No modelo clássico de Cidades Inteligentes, os sistemas ganham inteligência a partir de elementos digitais e são utilizados por seres humanos. Neles, podem ser aplicados os mais diversos sensores digitais como, por exemplo, sensores de movimento e posicionamento, e as pessoas agem como receptores e tomadores de decisão das informações geradas. No entanto, percebe-se que, assim como sensores digitais podem adquirir, analisar e modelar dados, seres humanos também são capazes de fazê-lo, sendo pois possível inserir as pessoas como elementos mais participativos no sistema e na cidade.

Diante disto, os sistemas passam a apoiar-se no conceito de crowdsourcing "uso das habilidades perceptuais e cognitivas de um grupo de indivíduos para resolver um problema" (Erickson, 2010) - utilizando-se de sensores humanos (Crowd Sensors), apoiando-se na premissa de que, ainda segundo Erickson, "humanos podem contribuir com conhecimento qualitativo mais profundo, analisar dados vagos ou incompletos e agir de uma forma em que sistemas digitais frequentemente não são capazes".

Nesta pesquisa, deseja-se criar um modelo de Cidades Inteligentes utilizando Sensores Humanos visando propiciar um ambiente com suporte a melhorias na governança das cidades por meio de participação ativa da comunidade, de sorte a reportar problemas e acompanhar a aplicação das soluções propostas pelas autoridades.

O objetivo principal, no entanto, é a realização de uma análise da efetividade deste tipo de sensor no contexto descrito. Para tal fim, será desenvolvida uma ferramenta com base no modelo proposto e esta será disponibilizada para uso da população por um período de tempo. Em seguida, com os dados coletados do uso da 
ferramenta e com base em entrevistas realizadas com os usuários, será avaliada a efetividade dos sensores considerando algumas métricas detalhadas mais à frente e analisados os resultados considerando também o perfil social dos autores.

\section{Trabalhos Relacionados}

Atualmente, existe um número considerável de modelos propostos por pesquisadores no contexto de Cidades Inteligentes devido aos recentes avanços na computação ubíqua e à própria evolução tecnológica. Governos e grandes empresas estão participando da iniciativa e investindo em pesquisas no setor, buscando propor abordagens para melhorar a qualidade de vida dos cidadãos, a administração da infraestrutura e dos recursos disponíveis, reduzindo o impacto ambiental.

Os trabalhos de Harrison e Donnelly (Harrison et al., 2011), e também de Helal (2011), discutem sobre os aspectos e atividades na área de Cidades Inteligentes, o papel da Tecnologia da Informação (TI) no desenvolvimento de novas normas de comportamento para facilitar o crescimento das cidades e os benefícios que esta estratégia pode trazer para os governos, as organizações e os cidadãos, evidenciando sua importância no mundo atual. São mencionadas suas aplicações em cenários diversos como o gerenciamento de energia, água e do tráfego e no auxílio à segurança pública.

Governos como o do Rio de Janeiro e o de Dubuque estão também investindo na área de Cidades Inteligentes para o desenvolvimento de soluções para alguns de seus problemas mais alarmantes (Naphade et al., 2011). No caso do Rio de Janeiro, as fortes chuvas que acontecem em todo mês de janeiro foram a maior motivação para a busca de soluções. Por terem como consequência graves desastres como alagamentos e deslizes de terra, e tendo em vista o risco em que sua população vive e os futuros eventos a serem sediados em seu território (como a Copa do Mundo de Futebol de 2014 e os Jogos Olímpicos de 2016), as autoridades do estado buscaram a IBM para implantação de um sistema para melhor administração das crises e do tráfego na cidade. No caso da cidade de Dubuque, em Iowa nos Estados Unidos, problemas na economia motivaram uma mudança no pensamento das autoridades para a implantação de um sistema de Cidades Inteligentes que provesse a otimização do consumo de água e energia, visando prosperidade econômica e integridade ambiental.

Portanto, pode-se perceber que nas mais diversas áreas estão sendo aplicadas soluções de Cidades Inteligentes, principalmente utilizando sensores digitais. Porém, uma das maiores dificuldades encontradas para a implantação de grande parte das soluções propostas é a falta de ambientes reais com o suporte necessário para a sua execução, como a falta dos sensores necessários cobrindo todo o território alvo.

Quanto a soluções utilizando sensores humanos é encontrado um número reduzido de estudos na literatura. Em sua pesquisa, Demirbas et al. (2010) fizeram uso destes sensores na forma de usuários do microblog Twitter. Seu protótipo desenvolvido tinha como objetivo o repasse de consultas sobre as condições climáticas a diversos usuários da rede social e, em seguida, retornava o resultado ao usuário que as submeteu. A análise feita pelos autores foi apenas a realização de comparações dos resultados obtidos com dados oficiais sobre o clima, considerando três cidades diferentes, o horário do dia em que as respostas eram submetidas e também o cliente utilizado para responder 
às consultas (cliente móvel ou $\mathrm{PC}$ ). $\mathrm{O}$ maior problema encontrado nesta abordagem, porém, é seu escopo reduzido.

Brabham (2010) também fez uso de sensores humanos e focou seu estudo na análise da efetividade de tais sensores no contexto da participação pública em atividades de planejamento da cidade. $\mathrm{O}$ autor submeteu um problema (no caso o projeto de uma parada de ônibus) para que a comunidade postasse ideias e votasse nos modelos uns dos outros a fim de eleger o melhor. Neste contexto, o autor realizou uma análise qualitativa por meio de entrevistas com os usuários da ferramenta.

Este estudo pretende fazer uso dos sensores humanos uma vez que, além de serem mais baratos que os digitais, possuem maior potencial e possibilidade de contribuição mais generalizada (sobre uma maior gama de domínios) para, em seguida, realizar uma avaliação da sua efetividade no contexto de Cidades Inteligentes.

\section{Crowd4City: Modelo e Arquitetura do Sistema}

No trabalho proposto, a ferramenta desenvolvida terá como objetivo propiciar um ambiente em que a população possa participar mais ativamente da gestão da cidade, oferecendo esta interação por meio de uma rede social a ser criada.

Inicialmente, será realizada a recuperação das informações relevantes a partir dos usuários da rede social com relação a tópicos diversos como, por exemplo, pontos com altos índices de criminalidade, deformações nas ruas e estradas e locais com problemas na rede de saneamento básico. Desta forma, os problemas passarão a estar registrados em um meio público, podendo sofrer uma repercussão maior, e a população poderá realizar uma cobrança maior das autoridades. Em seguida, estas informações serão analisadas a fim de avaliar a efetividade dos sensores humanos neste contexto.

\subsection{Modelo}

$\mathrm{Na}$ ferramenta, serão utilizadas tags normais e geográficas (separadamente ou combinadas) para anotação dos recursos e publicações feitas pelos usuários. Para a modelagem e representação serão utilizadas e estendidas ontologias já existentes, como FOAF (Friend of a Friend) e SIOC (Breslin et al., 2005). Os usuários da ferramenta, portanto, servirão como sensores criando publicações e anexando arquivos multimídia com informações sobre a cidade, possibilitando que comentários possam ser adicionados por outros usuários.

Um problema encontrado nesta abordagem é a susceptibilidade a erros que estas entradas humanas podem apresentar, podendo gerar spam e conteúdo indesejado para o sistema. Para contorná-lo, é proposta a criação de uma hierarquia de mediação dos usuários: de acordo com as avaliações que receberem através de suas publicações, passarão a ser classificados em uma das categorias da hierarquia a ser criada. Inicialmente, a hierarquia irá considerar a localização das pessoas e os assuntos sobre os quais suas publicações possuírem mais qualificações positivas.

Desta maneira, espera-se reduzir o número de publicações de conteúdo inadequado ou que, por ventura, possuam algum tipo de viés (político, por exemplo) envolvendo pessoas de diferentes classes e pontos de vista no processo de publicação. Todas as postagens na rede social deverão receber o parecer de um mediador para serem 
aprovadas e, caso os usuários que tiverem suas publicações recusadas acreditarem ter sofrido um mau julgamento, estes poderão ainda recorrer aos níveis mais altos da hierarquia.

Outro desafio presente na abordagem sugerida é a necessidade de algum tipo de mecanismo que incentive os cidadãos a fazerem uso da ferramenta. Para tanto, a parceria com organizações como o Observatório Social do Nordeste (já firmada) será de grande importância, uma vez que esta irá apoiar-se na ferramenta e promoverá seu uso por meio de mobilizações e seminários que serão realizados.

\subsection{Arquitetura}

Nesta seção é apresentada a arquitetura em três camadas do sistema Crowd4City. Conforme ilustrado na Figura 1 (a), o usuário fará o acesso à ferramenta utilizando um browser HTTP, executando as páginas JSP que irão compor a interface. A Camada de Apresentação contém os elementos da aplicação que serão visualizados pelos usuários finais e que serão utilizados para a inserção das informações advindas dos sensores humanos. Além das páginas JSP, a interface utilizará scripts na linguagem Javascript para realizar o acesso aos serviços externos do Google Maps, usado para exibição do mapa e recuperação de informações geográficas de determinados locais.

A Camada de Negócios é responsável pelo processamento das operações requisitadas pelos usuários e contém toda a lógica de negócio do sistema desenvolvido. Como pode ser observado na Figura 1 (b), a lógica de negócios será composta por sete módulos principais: os gerenciadores do sistema, dos sensores humanos, dos usuários, de recursos multimídia, das tags, e os serviços de persistência da informação e de descoberta da informação.

Na Camada de Dados - Figura 1 (c) - encontram-se os dados que serão processados e/ou gerados pela camada de negócios e visualizados pelos usuários na Camada de Apresentação, utilizando três repositórios de dados: o primeiro para armazenamento de toda informação da rede social e suas interações; o segundo como repositório dos arquivos multimídia compartilhados pelos usuários; e o último utilizado para armazenar os metadados geográficos e executar as funções geográficas do PostGIS.

\section{Trabalhos Futuros}

Com a ferramenta do estudo em mãos, o próximo passo será disponibilizá-la para uso em ambientes reais. Será feita sua implantação em uma cidade considerada de porte médio e caráter urbano (Campina Grande, Paraíba), e também em um ambiente menor e de população mais consciente nos quesitos de preservação do meio ambiente e manutenção da infraestrutura - o município de Xapuri, no estado do Acre.

Uma vez colhidos os dados da execução da ferramenta, serão realizados diversos testes estatísticos para avaliar a efetividade dos sensores humanos no âmbito de Cidades Inteligentes. Serão analisadas métricas como a razão de rejeição de publicações, a porcentagem daquelas que obtiveram feedback, a porcentagem de publicações que necessitaram passar por cada um dos níveis da hierarquia e também serão feitas análises com relação ao perfil social dos usuários (sexo, faixa etária, localização e classe social). A fim de obter suas impressões sobre a efetividade e a eficiência da solução, serão realizadas ainda entrevistas com os moradores das cidades. 
Buscando alcançar um número maior de usuários e também um impacto mais amplo, será estudada a possibilidade de implementar e implantar um plugin da ferramenta em algumas das redes sociais mais utilizadas atualmente, como o Facebook.

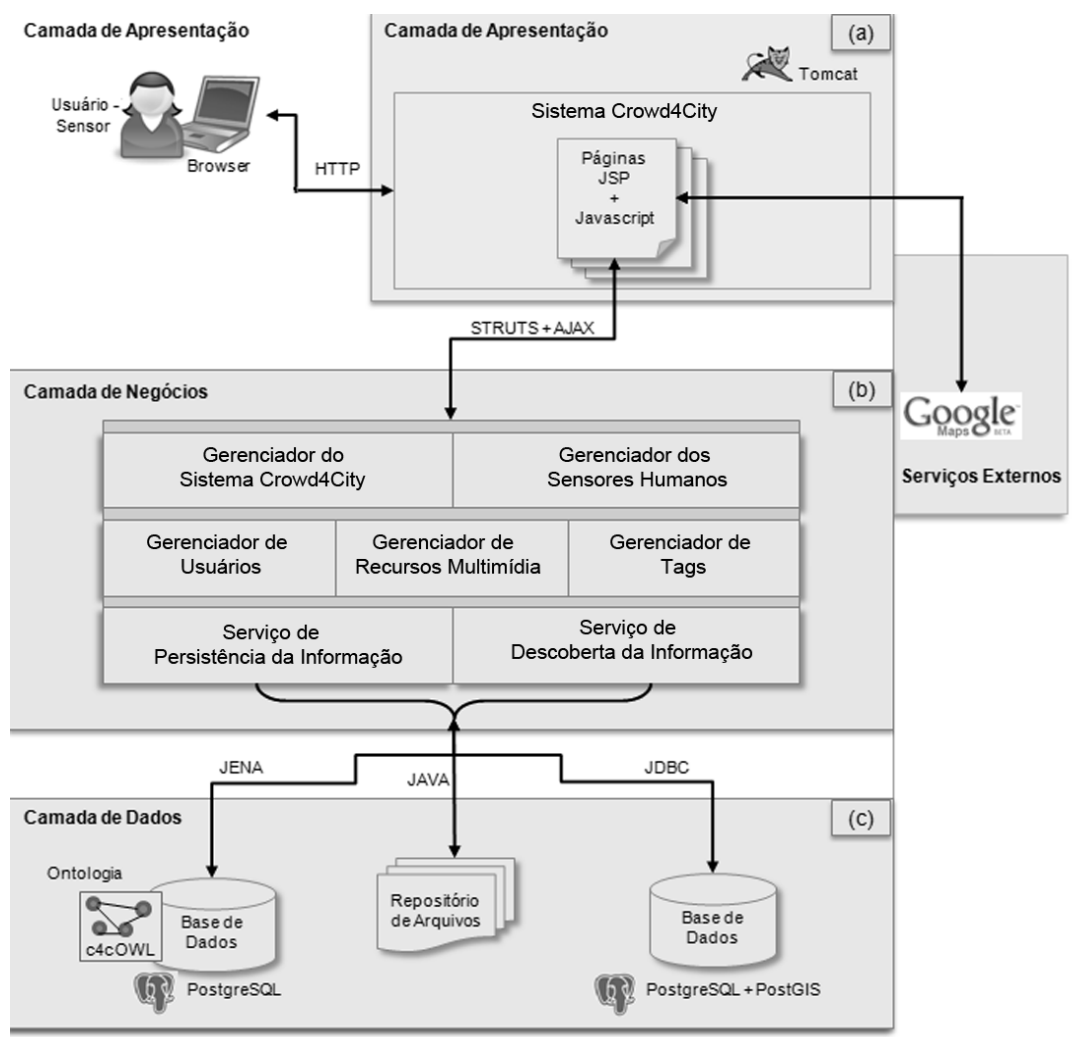

Figura 1. Arquitetura em três camadas do sistema Crowd4City

\section{Referências Bibliográficas}

Brabham, D. C. (2010). "The Effectiveness of Crowdsourcing Public Participation in a Planning Context”, Social Science Research Network, vol. 85, no.9, p. S424-9.

Breslin, J. G., Harth, A., Bojars, U. and Decker, S. (2009). "Towards Semantically Interlinked Online Communities", 2nd European Semantic Web Conference, Heraklion, Greece, p. 500-514.

Demirbas, M., Bayir, M. A., Akcora, C. G., Yilmaz, Y. S. and Ferhatosmanoglu, H. (2010). "Crowd-Sourced Sensing and Collaboration Using Twitter", In: IEEE International Symposium on a World of Wireless, Mobile and Multimedia Networks.

Erickson, T. (2010). "Geocentric Crowdsourcing and Smarter Cities: Enabling Urban Intelligence in Cities and Regions", In: $12^{\text {th }}$ ACM International Conference on Ubiquituous Computing.

Harrison, C. and Donnelly, I. A., “A Theory of Smart Cities”, Cities, July 2011, p. 1-15.

Helal, S. (2011). "IT Footprinting - Groundwork for Future Smart Cities", Computer, vol. 44, no.6, p. 30-31.

Naphade, M., Banavar G., Harrison, C., Paraszczak, J. and Morris, R. (2011). "Smarter Cities and Their Innovation Challenges”, Computer, vol.44, no.6, p. 32-39. 